\title{
Maternal diabetes and incidence of childhood cancer - a nationwide cohort study and exploratory genetic analysis
}

This article was published in the following Dove Press journal:

Clinical Epidemiology

\section{Anna Deleskogl,* \\ Marcel den Hoed ${ }^{2,3, *}$ \\ Giorgio Tettamanti' \\ Sofia Carlsson' \\ Rickard Ljung' \\ Maria Feychting' \\ Hannah L Brooke'}

'Unit of Epidemiology, Institute of Environmental Medicine, Karolinska Institutet, Stockholm, ${ }^{2}$ Department of Immunology, Genetics and Pathology, ${ }^{3}$ Science for Life Laboratory, Uppsala University, Uppsala, Sweden

*These authors contributed equally to this work
Correspondence: Hannah L Brooke Unit of Epidemiology, Institute of Environmental Medicine, Karolinska Institutet, PO Box 210, Stockholm 171

77, Sweden

Tel +46852487537

Email hannah.brooke@ki.se
Background: The etiology of childhood cancer is not well understood, but may be linked to prenatal and perinatal factors, such as maternal diabetes. However, this association has not been examined in depth. We aimed to determine if maternal diabetes is associated with risk of childhood brain tumor (CBT), leukemia (all types combined and acute lymphoblastic leukemia [ALL] separately), and lymphoma.

Methods: All children born in Sweden between 1973 and $2014(n=4,239,965)$ were followed from birth until first cancer diagnosis, age 15 years, or December 31, 2015. Data on maternal diabetes, childhood cancer, and covariates were obtained from nationwide health registers. Incidence rate ratios (IRRs) and 95\% confidence intervals (CIs) were calculated using Cox regression adjusted for potential confounders/mediators. Additionally, we performed an exploratory analysis using results from published genome-wide association studies and functional annotation.

Results: Maternal diabetes was associated with lower risk of CBT (adjusted IRR [95\% CI]: 0.56 [0.35-0.91]) and higher risk of leukemia (adjusted IRR: 1.47 [1.13-1.92] for all leukemia combined and 1.64 [1.23-2.18] for ALL). These associations were similar for both maternal type 1 diabetes and gestational diabetes. Associations of five previously identified genetic loci were compatible with a causal effect of diabetes traits on neuroblastoma and common Hodgkin's lymphoma.

Conclusion: Children whose mother had diabetes had lower risk of CBT and higher risk of leukemia, compared with children whose mother did not have diabetes. Our results are compatible with a role of prenatal and perinatal glycemic environment in childhood cancer etiology.

Keywords: neoplasm, childhood, diabetes, fetal growth, perinatal environment

\section{Introduction}

Cancer is the second most common cause of death in children in high-income countries, with incidence rates in Europe increasing by around 1\% per year. ${ }^{1}$ Although the etiology of childhood cancer is not well understood, the typically early onset suggests that genetic, prenatal, and perinatal factors may be involved. ${ }^{2,3}$

Perinatal factors linked to gestational diabetes, such as high fetal growth and high birth weight, have been associated with increased risk of childhood brain tumor (CBT) and leukemia. ${ }^{4-6}$ As such, maternal diabetes may influence the risk of childhood cancer. $^{7-10}$ The largest study to date showed an increased risk of leukemia, particularly acute lymphoblastic leukemia (ALL), and Wilms tumor among children of mothers with pre-pregnancy diabetes, but not gestational diabetes. ${ }^{7}$ However, other studies have typically been small ${ }^{8,9}$ and have not examined associations with cancer subtypes. ${ }^{8-10}$ 
In adults, type 1 and type 2 diabetes are both associated with cancer risk, with the direction of association varying by cancer subtype. ${ }^{11-13}$ By examining if such differences are also present in children exposed to maternal diabetes, we may acquire insights into etiological differences across cancer subtypes. By additionally examining associations of cancer risk with specific types of maternal diabetes, we may increase our understanding of the role of hyperglycemia and autoimmunity in childhood cancer risk.

In this nationwide Swedish cohort study, we examined the association of maternal diabetes and diabetes subtypes with the most common types of childhood cancer: CBT, leukemia (all types combined and the most common subtype, ALL, separately), and lymphoma.

\section{Methods Study cohort}

We conducted a population-based cohort study of all live births in Sweden between 1973 and 2014, identified from the Swedish Medical Birth Register. This register contains information on birth and perinatal factors for $98 \%$ of births in Sweden since $1973 .{ }^{14}$ Children were followed from birth and censored at death, emigration, first primary cancer diagnosis, their 15th birthday, or end of follow-up (December 31, 2015). Date of death and migration were ascertained from the Cause of Death Register and the Migration Register, respectively. All cancer diagnoses between 1973 and 2015 were obtained from the nationwide Cancer Register, which has an overall completeness of at least $96 \% .{ }^{15}$ Children without sufficient information to enable their mother to be identified in the hospital inpatient and outpatient registers $(n=5,237)$ were not included in this study. In total, $<1 \%$ of the study population had missing data on at least one covariate $(n=37,354)$, leaving 4,239,965 children for inclusion in the analyses. Ethical approval for the study was granted by the Regional Ethical Review Board, Stockholm, Sweden (2011/634-31/4). Informed consent was not required for this study since it was based on de-identified data from national registers and publically available data. Patient data confidentiality was assessed and approved by the National Board of Health and Welfare and the Ethical Review Board before de-identified personal data were disclosed to the research team.

\section{Exposure ascertainment}

Mothers with diabetes diagnosed prior to and during pregnancy were identified using International Statistical Classification of Disease and Related Health Problems (ICD) codes in the Medical Birth Register and hospital inpatient and outpatient registers, and an indicator variable (yes/no) for maternal diabetes in the Medical Birth Register (ICD codes described in Table S1A). Since type 2 diabetes is typically preceded by a period of prediabetes, we also included mothers with type 2 diabetes diagnoses up to 5 years after the child's birth as exposed. For the main analyses, all diabetes diagnoses were pooled. However, type of maternal diabetes (type 1 , type 2 , or gestational diabetes) was subsequently categorized, as described in Table S1B.

\section{Outcome ascertainment}

Cases of childhood cancer were defined as the first primary cancer diagnosis in the Cancer Register during the follow-up period. Cancer diagnoses were identified using the following ICD-7 codes (ICD-8 for leukemia), which were available throughout the follow-up period: CBT, 193.0; lymphoma, 200-202; leukemia (all subtypes types combined), 204-207; and ALL (the most common subtype of childhood leukemia, separately), 204.0. For other cancer subtypes, an insufficient number of exposed cases were available to calculate meaningful risk estimates.

\section{Statistical analysis}

Cox proportional hazards models were used to estimate incidence rate ratios (IRRs) and 95\% confidence intervals $(95 \%$ CIs) of all cancer subtypes combined, CBT, all leukemia subtypes combined, ALL, and lymphoma. Children born to mothers without diabetes were the reference group. Mother's identification number was used as a clustering variable, and robust standard errors were estimated to account for lack of independence between siblings. In model 1, unadjusted estimates are presented. Model 2 was adjusted for potential confounders: child's sex, decade of birth, maternal age at child's birth, highest attained parental education level, and birth order. Model 3 was additionally adjusted for birth weight by gestational age, gestational age, child's diabetes status, and birth defects in the child. While these factors may be considered mediators rather than confounders, model 3 was designed to isolate the association with maternal diabetes itself. Categorization and ICD codes used for all covariates are described in Table 1. We used the same aforementioned statistical methods to examine associations of maternal type 1 , type 2 , and gestational diabetes with offspring cancer separately. All analyses were performed in Stata version 13.1 (StataCorp, 2013).

\section{Additional analyses}

Additional analyses were conducted in a sub-sample of the cohort further adjusting model 2 and model 3 for maternal 
Table I Potential confounders included as covariates in main analyses and additional analysis

\begin{tabular}{|c|c|c|c|}
\hline & Covariate & Register & Categories and ICD codes \\
\hline \multirow{11}{*}{ 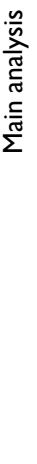 } & Maternal age at child's birth & Medical Birth Register & Continuous \\
\hline & Birth order & Medical Birth Register & $\mathrm{I}, 2, \geq 3$ \\
\hline & Child's sex & Medical Birth Register & Male, female \\
\hline & Birth weight by gestational age & Medical Birth Register & SGA (birth weight below the 10th percentile for gestational age) \\
\hline & & & AGA \\
\hline & & & LGA (birth weight above the 90th percentile for gestational age) \\
\hline & Highest attained parental education level & LISA/census in $1970^{a}$ & Primary (less than 10 years of education) \\
\hline & & & Secondary ( $10-12$ years of education) \\
\hline & & & Tertiary (more than 12 years of education) \\
\hline & $\begin{array}{l}\text { Child's diabetes diagnoses before cancer } \\
\text { diagnosis or before age } 15 \text { years }\end{array}$ & $\begin{array}{l}\text { Swedish hospital inpatient } \\
\text { register }\end{array}$ & ICD-8 and ICD-9, 250; ICD-I0, EI0, and EII \\
\hline & Birth defects & & ICD-9: 740-759; ICD-I0: Q00-Q99 \\
\hline \multirow{7}{*}{ 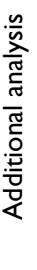 } & Maternal smoking during pregnancy ${ }^{\mathrm{b}}$ & Medical Birth Register & None \\
\hline & & & I-9 cigarettes/day \\
\hline & & & $\geq 10$ cigarettes/day \\
\hline & Pre-pregnancy BMIb & Medical Birth Register & Underweight, $\mathrm{BMI}<18.5 \mathrm{~kg} / \mathrm{m}^{2}$ \\
\hline & & & Healthy weight, BMI $18.5-<25 \mathrm{~kg} / \mathrm{m}^{2}$ \\
\hline & & & Overweight, BMI $25-<30 \mathrm{~kg} / \mathrm{m}^{2}$ \\
\hline & & & Obese, $\mathrm{BMI} \geq 30 \mathrm{~kg} / \mathrm{m}^{2}$ \\
\hline
\end{tabular}

Notes: aHighest attained parental education was taken from LISA for all parents included in LISA at any time between 1990 and 2014 . For parents who were not included in LISA during this period we used information on education from the census in 1970. 'Data only available from 1983 onwards.

Abbreviations: ICD, International Statistical Classification of Disease and Related Health Problems; SGA, small for gestational age; AGA, appropriate for gestational age; LGA, large for gestational age; LISA, The Longitudinal Integration Database for Health Insurance and Labor Market Studies; BMI, body mass index.

smoking during pregnancy and pre-pregnancy body mass index (BMI; data only available from 1983 onwards) (Table 1). Additional analyses were also conducted to assess associations of maternal diabetes with childhood cancer diagnoses up to age 6 years and age 20 years rather than age 15 years.

\section{Causal inference and functional annotation}

In order to identify mechanisms that may explain the associations we observed, we performed an exploratory genetic analysis using results from publically available genomewide association studies (GWAS) for diabetes traits ${ }^{16-19}$ and cancer, ${ }^{20-22}$ as identified using the GWAS catalogue, ${ }^{23}$ SNiPA, ${ }^{24}$ and GRASP. ${ }^{25}$ We subsequently functionally annotated loci that showed associations with diabetes as well as cancer traits for roles in regulatory processes and tissue-specific gene expression using publicly available bioinformatics tools. ${ }^{26,27}$ The process for the exploratory analysis of GWAS and functional annotation to examine causal inference and molecular mechanisms is illustrated in Figure $1 .{ }^{23-27} \mathrm{~A}$ formal Mendelian randomization study would likely be underpowered due the small number of cases for each of the cancer subtypes, both in the observational and genetic association studies. However, the exploratory analysis described here may still provide useful insights regarding causal inference, and provide a first step towards functional follow-up experiments.
Additional details about classification of maternal diabetes type and assessment of proportional hazards assumptions are available in Supplementary materials.

\section{Results}

In total, $61,212(1.4 \%)$ children had a mother with a diabetes diagnosis. Mothers with diabetes were slightly older and had a higher pre-pregnancy BMI than mothers without diabetes (Table 2). Children whose mother had diabetes were more likely to have diabetes themselves, be the 3 rd or later child, or be born large for gestational age, while they were less likely to be born after 40 weeks of gestation (Table 2).

In 51,864,010 person-years of follow-up time, 8,839 cases of childhood cancer were identified. Mean (SD) age at diagnosis was 6.0 (4.4) years and $46.2 \%$ of cases were girls.

We found no association between mothers' diabetes status and cancer in their children when all cancer subtypes were combined (Table 3). For specific cancer subtypes, however, children of mothers with diabetes had a $41 \%$ lower risk of CBT compared with children of mothers without diabetes (IRR [95\% CI]: 0.59 [0.36, 0.96]). The association remained after adjusting for the child's sex, decade of birth, maternal age, highest attained parental education level, birth order, birth weight by gestational age, gestational age, child's diabetes status, and child birth defects (IRR [95\% CI]: 0.56 [0.35, $0.91]$ ). By contrast, children of mothers with diabetes had a 47\% higher risk of all types of leukemia combined (IRR 


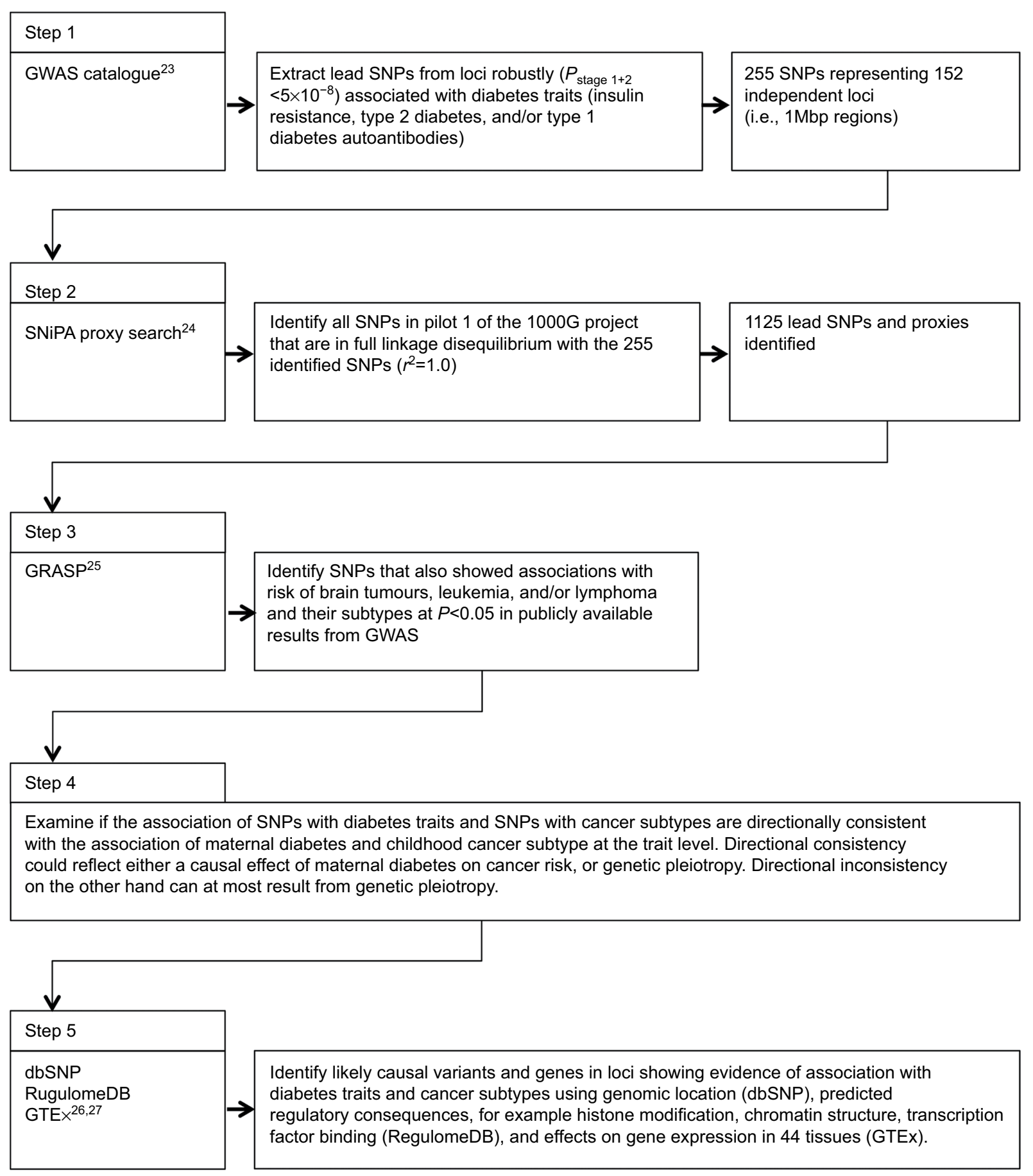

Figure I Flow chart of exploratory analysis of GWAS and functional annotation to examine causal inference and molecular mechanisms. Abbreviation: GWAS, genome-wide association studies.

[95\% CI]: $1.47[1.13,1.92])$, and a $64 \%$ higher risk of ALL than children of mothers without diabetes (IRR [95\% CI]: $1.64[1.23,2.18])$, in adjusted models. The adjusted IRR for lymphoma was $1.45(0.90,2.36)$, suggesting a higher incidence rate in children of mothers with diabetes than children of mothers without diabetes.
Results from analyses examining subtypes of maternal diabetes indicate a lower risk of CBT among children of mothers with type 1 diabetes and gestational diabetes (Table 4). There was a higher risk of leukemia and ALL among children of mothers with type 1 diabetes and gestational diabetes (Table 4). This association was stronger 
Table 2 Accumulated person-years at risk, demographic characteristics, and birth characteristics of children born in 1973-2014 and followed from birth for up to 15 years or to December 2015, according to maternal diabetes status

\begin{tabular}{|c|c|c|}
\hline Characteristics & $\begin{array}{l}\text { Children of mothers without } \\
\text { diabetes }^{a}(n=4,178,753)\end{array}$ & $\begin{array}{l}\text { Children of mothers with } \\
\text { diabetes }^{a}(n=6 I, 2 \mid 2)\end{array}$ \\
\hline Person-years at risk & $51,188,995$ & 675,015 \\
\hline Mothers age at child's birth (years), mean (SD) & $28.7(5.2)$ & $30.9(5.5)$ \\
\hline \multicolumn{3}{|l|}{ Mother's diabetes type } \\
\hline Type I diabetes & & $248,709(36.8)$ \\
\hline Type 2 diabetes & & $|32,96|(19.7)$ \\
\hline GDM & & $293,345(43.5)$ \\
\hline \multicolumn{3}{|l|}{ Child's diabetes status } \\
\hline No & $50,944,404(99.5)$ & $642,315(95.2)$ \\
\hline Yes & $244,591(0.5)$ & $32,700(4.8)$ \\
\hline \multicolumn{3}{|l|}{ Child's sex } \\
\hline Boys & $26,289,766(51.4)$ & $348,774(51.7)$ \\
\hline Girls & $24,899,229(48.6)$ & $326,241(48.3)$ \\
\hline \multicolumn{3}{|l|}{ Birth order } \\
\hline I & $21,445,817(4 \mid .9)$ & $237,496(35.2)$ \\
\hline 2 & $18,746,963(36.6)$ & $232,066(34.4)$ \\
\hline $3+$ & $10,996,215(21.5)$ & $205,453(30.4)$ \\
\hline \multicolumn{3}{|l|}{ Birth weight by gestational age } \\
\hline SGL & $5,158,361(10.1)$ & $46,553(6.9)$ \\
\hline AGA & $40,927,017(80.0)$ & $434,737(64.4)$ \\
\hline LGA & $5,103,616(10.0)$ & $193,725(28.7)$ \\
\hline \multicolumn{3}{|l|}{ Gestational age } \\
\hline$<37$ weeks & $2,802,759(5.5)$ & $93,337(13.8)$ \\
\hline $37-40$ weeks & $34,720,592(67.8)$ & $501,756(74.3)$ \\
\hline$>40$ weeks & $13,665,644(26.7)$ & $79,922(11.8)$ \\
\hline \multicolumn{3}{|l|}{ Birth defects } \\
\hline No & $49,155,526(96.0)$ & $643,277(95.3)$ \\
\hline Yes & $2,033,469(4.0)$ & $31,738(4.7)$ \\
\hline \multicolumn{3}{|l|}{ Highest attained parental education level } \\
\hline Primary (<10 years) & $2,977,053(5.8)$ & $45,769(6.8)$ \\
\hline Secondary ( $10-12$ years) & $23,006,602(44.9)$ & $323,052(47.9)$ \\
\hline Tertiary (>12 years) & $25,205,339(49.2)$ & $306,194(45.4)$ \\
\hline \multicolumn{3}{|l|}{ Birth decade } \\
\hline $1973-1980$ & I0,04I,403 (19.6) & $44,947(6.7)$ \\
\hline $1980-1990$ & $14, \mid 49,956(27.6)$ & $154,272(22.9)$ \\
\hline $1990-2000$ & $|4,89|, \mid 62(29.1)$ & $234,946(34.8)$ \\
\hline $2000-2010$ & $10,804,766(21.1)$ & $211,238(31.3)$ \\
\hline $2000-2014$ & $\mathrm{I}, 30 \mathrm{I}, 709(2.5)$ & $29,612(4.4)$ \\
\hline Maternal pre-pregnancy BMI $\left(\mathbf{k g} / \mathbf{m}^{2}\right)$, mean (SD) & $23.8(4.2)$ & $27.3(5.9)$ \\
\hline \multicolumn{3}{|l|}{ Maternal smoking during pregnancyc } \\
\hline 0 cigarettes/day & $29,053,180(81.1)$ & $463,682(81.9)$ \\
\hline I-9 cigarettes/day & $4,367,74 I(\mid 2.2)$ & $63,212(11.2)$ \\
\hline $10+$ cigarettes/day & $2,400,719(6.7)$ & $39,212(6.9)$ \\
\hline
\end{tabular}

Notes: a Data shown as $\mathrm{n}(\%)$ unless otherwise specified. ${ }^{\mathrm{b}}$ Among mothers included in the main analysis with this information available ( $\left.\mathrm{n}=2,596,189\right)$. ${ }^{\mathrm{c}} \mathrm{Among}$ mothers included in the main analysis with this information available $(n=3,154,313)$.

Abbreviations: GDM, gestational diabetes mellitus; SGA, small for gestational age; AGA, appropriate for gestational age; LGA, large for gestational age; BMI, body mass index.

among children of mothers with type 1 diabetes. There was no evidence of an association of maternal type 2 diabetes with CBT, leukemia, or ALL. However, relatively few mothers had a type 2 diabetes diagnosis and hence few cases of cancer were identified in children of this subgroup of women (Table 4). For lymphoma point estimates indicate a positive association with type 2 diabetes and gestational diabetes. However, there were few exposed cases, which led to wide CIs for these estimates (Table 4).

Analyses with additional adjustment for pre-pregnancy BMI and maternal smoking during pregnancy (available for a subset of mothers) yielded largely similar results as 
Table 3 IRRs and 95\% Cls of different types of childhood cancer by maternal diabetes status in 4,239,965 children born in I973-20I4 and followed from birth for up to age 15 years or to December 2015

\begin{tabular}{|c|c|c|c|c|}
\hline $\begin{array}{l}\text { Outcome and exposure } \\
\text { categories }\end{array}$ & $\begin{array}{l}\text { Number of } \\
\text { cases }\end{array}$ & $\begin{array}{l}\text { Model Ia IRR } \\
(95 \% \mathrm{CI})\end{array}$ & $\begin{array}{l}\text { Model } 2^{\mathrm{b}} \text { IRR } \\
(95 \% \mathrm{CI})\end{array}$ & $\begin{array}{l}\text { Model 3c IRR } \\
(95 \% \mathrm{CI})\end{array}$ \\
\hline \multicolumn{5}{|c|}{ All cancer subtypes combined } \\
\hline No maternal diabetes & 8,709 & Reference & Reference & Reference \\
\hline Maternal diabetes & 130 & I.II $(0.94,1.32)$ & $1.10(0.92,1.30)$ & $1.06(0.89,1.26)$ \\
\hline \multicolumn{5}{|l|}{ СВТ } \\
\hline No maternal diabetes & 2,063 & Reference & Reference & Reference \\
\hline Maternal diabetes & 16 & $0.59(0.36,0.96)$ & $0.59(0.36,0.97)$ & $0.56(0.35,0.91)$ \\
\hline \multicolumn{5}{|l|}{ Leukemia $^{d}$} \\
\hline No maternal diabetes & 2,631 & Reference & Reference & Reference \\
\hline Maternal diabetes & 56 & $1.57(1.20,2.04)$ & $1.52(1.16,1.98)$ & $1.47(1.13,1.92)$ \\
\hline \multicolumn{5}{|l|}{ ALL } \\
\hline No maternal diabetes & 2,043 & Reference & Reference & Reference \\
\hline Maternal diabetes & 48 & $1.73(1.30,2.30)$ & $1.67(1.25,2.22)$ & $1.64(1.23,2.18)$ \\
\hline \multicolumn{5}{|l|}{ Lymphoma } \\
\hline No maternal diabetes & 884 & Reference & Reference & Reference \\
\hline Maternal diabetes & 17 & $1.48(0.92,2.39)$ & $\mathrm{I} .43(0.88,2.3 \mathrm{I})$ & $1.45(0.90,2.36)$ \\
\hline
\end{tabular}

Notes: Estimated using Cox proportional hazards models. a Unadjusted estimates. ${ }^{b}$ Adjusted for child's sex, decade of birth, maternal age, highest attained parental education level, and birth order. 'Adjusted for child's sex, decade of birth, maternal age, highest attained parental education level, birth order, birth weight by gestational age, gestational age, child's diabetes status, and child birth defects. 'All types of leukemia combined.

Abbreviations: IRRs, incidence rate ratios; Cls, confidence intervals; CBT, childhood brain tumor; ALL, acute lymphoblastic leukemia.

compared with the main analysis (Table S2). However, in this subset of children there was no evidence of an association between maternal diabetes and lymphoma either before or after additionally adjusting for maternal smoking and BMI. The second additional analysis assessed associations with cancer diagnosed before age 6 years. The results were consistent with the main findings and suggest stronger associations in early childhood (Table S3). In one further additional analysis, extending follow-up to 20 years of age attenuated the associations of maternal diabetes with CBT, while the association with lymphoma was strengthened compared with results from the main analysis (Table S4).

Examining the association of loci identified by large-scale GWAS for diabetes traits $\left(P<5 \times 10^{-8}\right),{ }^{16-19}$ with risk of cancer subtypes $(P<0.05)$ identified 13 independent loci that showed suggestive evidence of association with risk of neuroblastoma (ten loci), ${ }^{20}$ common Hodgkin lymphoma (one locus), ${ }^{21}$ or childhood ALL (two loci) (Table S5). ${ }^{22}$ No evidence of associations with other cancer subtypes were observed at $P<0.05$. Of the ten neuroblastoma-associated loci, an interaction with BMI for fasting insulin obscured the direction of effect for one locus. Of the remaining nine loci, four showed directionally contrasting associations with the risk of insulin resistance and diabetes on one hand and neuroblastoma on the other hand, in line with the inverse association between maternal diabetes and risk of CBT (Table S5). The effect of these four loci is thus compatible with a causal (protective) effect of maternal insulin resistance and type 2 diabetes on the risk of neuroblastoma. Two of the four loci stand out in terms of biological relevance. Firstly, the $T$ allele of rs 5215 - a missense variant in $K C N J 11$ - is associated with a lower risk of type 2 diabetes $\left(P=3 \times 10^{-11}\right),{ }^{18}$ a higher risk of neuroblastoma $(P=0.014)$ (Table $\mathrm{S} 5),{ }^{20}$ a lower expression of $K C N J 11$ in skeletal muscle $\left(P=3.2 \times 10^{-3}\right)$, and a trend for a lower expression of KCNJ11 in cortex, hypothalamus, and cerebellum $(P=0.016-0.032)$ (Table S6) ${ }^{27}$ Secondly, the A allele of rs308971 - an intronic variant in SYN2 - is associated with lower fasting insulin levels $\left(P=2 \times 10^{-7}\right),{ }^{16}$ a higher risk of neuroblastoma $(P=0.031)$ (Table $S 5),{ }^{20}$ and a lower expression of SYN2 in pancreas $\left(P=2.3 \times 10^{-8}\right)$, tibial artery $\left(P=8.4 \times 10^{-9}\right)$, and thyroid $\left(P=1.3 \times 10^{-7}\right)$ (Table S6). ${ }^{27}$ Among the five loci for which the diabetes-increasing allele is associated with an increased risk of neuroblastoma, which can by definition at most represent genetic heterogeneity, is an intronic variant in insulin-like growth factor 2 (IGF2) (rs1004446) for which the $\mathrm{G}$ allele is associated with the presence of type 1 diabetes autoantibodies $\left(P=3.0 \times 10^{-23}\right),{ }^{17}$ an increased risk of neuroblastoma $\left(P=8.8 \times 10^{-3}\right),{ }^{20}$ and a lower expression of $I G F 2$ in esophagus mucosa $\left(P=3.2 \times 10^{-5}\right) \cdot{ }^{27}$

The A allele of rs2476601 - an intronic variant in AP4B1$A S 1$ - has been associated with the presence of type 1 diabetes autoantibodies $\left(P=2.0 \times 10^{-111}\right)^{17}$ and increased risk of common Hodgkin's lymphoma $\left(P=3.7 \times 10^{-3}\right) \cdot{ }^{21} \mathrm{rs} 2476601$ is 
Table 4 IRRs and 95\% Cls of different types of childhood cancer by type of maternal diabetes in 4,239,965 children born in I973-20I4 and followed from birth for up to age 15 years or to December 2015

\begin{tabular}{|c|c|c|c|c|}
\hline $\begin{array}{l}\text { Outcome and exposure } \\
\text { categories }\end{array}$ & $\begin{array}{l}\text { Number of } \\
\text { cases }\end{array}$ & $\begin{array}{l}\text { Model Ia IRR } \\
(95 \% \mathrm{Cl})\end{array}$ & $\begin{array}{l}\text { Model 2b IRR } \\
(95 \% \mathrm{Cl})\end{array}$ & $\begin{array}{l}\text { Model 3c IRR } \\
(95 \% \mathrm{Cl})\end{array}$ \\
\hline \multicolumn{5}{|c|}{ All cancer subtypes combined } \\
\hline No maternal diabetes & 8,709 & Reference & Reference & Reference \\
\hline Type I diabetes & 53 & $1.24(0.95,1.63)$ & $1.24(0.94,1.62)$ & $1.18(0.90,1.55)$ \\
\hline Type 2 diabetes & 26 & $1.15(0.78,1.69)$ & $\mathrm{I} .13(0.77, \mathrm{I} .67)$ & $1.09(0.74,1.60)$ \\
\hline GDM & 51 & $0.99(0.75,1.30)$ & $0.96(0.73,1.27)$ & $0.94(0.72,1.24)$ \\
\hline \multicolumn{5}{|l|}{ СВТ } \\
\hline No maternal diabetes & 2,063 & Reference & Reference & Reference \\
\hline Type I diabetes & 5 & $0.50(0.21,1.20)$ & $0.50(0.21,1.20)$ & $0.45(0.19,1.08)$ \\
\hline Type 2 diabetes & 7 & $1.31(0.62,2.74)$ & $1.29(0.61,2.72)$ & $1.24(0.59,2.60)$ \\
\hline GDM & 4 & $0.34(0.13,0.90)$ & $0.34(0.13,0.91)$ & $0.34(0.13,0.90)$ \\
\hline \multicolumn{5}{|l|}{ Leukemia $^{d}$} \\
\hline No maternal diabetes & 2,631 & Reference & Reference & Reference \\
\hline Type I diabetes & 24 & $1.85(1.24,2.77)$ & $1.83(1.23,2.73)$ & $1.75(1.18,2.61)$ \\
\hline Type 2 diabetes & 8 & $1.17(0.58,2.34)$ & $1.14(0.57,2.28)$ & $1.10(0.55,2.20)$ \\
\hline GDM & 24 & $1.50(1.01,2.25)$ & $1.43(0.96,2.14)$ & $\mathrm{I} .4 \mathrm{I}(0.94,2.1 \mathrm{I})$ \\
\hline \multicolumn{5}{|l|}{ ALL } \\
\hline No maternal diabetes & 2,043 & Reference & Reference & Reference \\
\hline Type I diabetes & 21 & $2.09(1.36,3.21)$ & $2.06(1.34,3.17)$ & $2.02(1.33,3.08)$ \\
\hline Type 2 diabetes & 7 & $1.32(0.63,2.76)$ & $1.26(0.60,2.66)$ & $1.24(0.59,2.60)$ \\
\hline GDM & 20 & $\mathrm{I} .62(\mathrm{I} .04,2.5 \mathrm{I})$ & $1.53(0.98,2.38)$ & I.5I $(0.97,2.35)$ \\
\hline \multicolumn{5}{|l|}{ Lymphoma } \\
\hline No maternal diabetes & 884 & Reference & Reference & Reference \\
\hline Type I diabetes & 5 & $1.17(0.49,2.82)$ & $1.16(0.48,2.79)$ & $1.21(0.50,2.92)$ \\
\hline Type 2 diabetes & 4 & $1.74(0.65,4.65)$ & $1.64(0.61,4.39)$ & $1.64(0.61,4.40)$ \\
\hline GDM & 8 & $1.63(0.81,3.27)$ & $1.56(0.77,3.13)$ & $1.56(0.78,3.15)$ \\
\hline
\end{tabular}

Notes: Estimated using Cox proportional hazards models. aUnadjusted estimates. ${ }^{b}$ Adjusted for child's sex, decade of birth, maternal age, highest attained parental education level, and birth order. 'Adjusted for child's sex, decade of birth, maternal age, highest attained parental education level, birth order, birth weight by gestational age, gestational

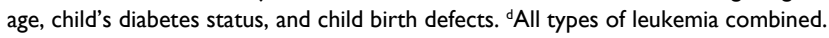

Abbreviations: IRRs, incidence rate ratios; Cls, confidence intervals; GDM, gestational diabetes mellitus; CBT, childhood brain tumor; ALL, acute lymphoblastic leukemia.

located in a binding site for the STAT3 transcription activator, ${ }^{26}$ which plays a key role in many cellular processes like cell growth and apoptosis. Mutations in STAT3 have been associated with infantile-onset multisystem autoimmune disease and hyper-immunoglobulin E (IgE) syndrome. ${ }^{28,29}$ Referrals for elevated IgE levels have previously resulted in a Hodgkin's lymphoma diagnosis. ${ }^{30}$

The $\mathrm{G}$ allele of rs7756992 - an intronic variant in CDKAL1 - has been associated with an increased risk of type 2 diabetes $\left(P=2 \times 10^{-26}\right)^{18}$ and an increased risk of childhood ALL $(P=0.012) .{ }^{22}$ rs 7756992 is located in a binding site for regulatory factor $\mathrm{X} 3$ (encoded by $R F X 3$ ), ${ }^{26}$ which influences HLA class II expression. HLA class II disparity is important for efficient induction of graft vs. leukemia immunity against poorly immunogenic ALL. ${ }^{31}$

\section{Discussion}

In this large nationwide study, children of mothers with diabetes had a lower risk of CBT and a higher risk of all leukemia subtypes combined, ALL, and lymphoma than children of mothers without diabetes. This study is the first to examine different types of maternal diabetes and specific subtypes of offspring cancer in detail. The association with CBT was found for both maternal type 1 diabetes and gestational diabetes. The associations with leukemia and ALL were most pronounced for maternal type 1 diabetes, with indication of the same direction of effect for gestational diabetes.

In contrast to previous studies, ${ }^{9,10}$ we found no association between maternal diabetes and offspring cancer risk when all cancer subtypes were combined. Compared with our study, previous studies were small, ${ }^{9}$ or followed children into adulthood, ${ }^{10}$ which limits the comparability. The etiology of different types of childhood cancer is likely to vary substantially and hence pooling all cancer subtypes may have spuriously hidden associations of cancer subtypes.

The results of our study are in line with those of a previous study that showed inverse associations of maternal pre-pregnancy diabetes with offspring risk of several types 
of CBT. ${ }^{7}$ In our study, this association was independent of the potential confounding/mediating factors, maternal and paternal age, highest attained parental education level, birth order, birth weight by gestational age, gestational age, child's sex, and child's diabetes status. Moreover, the association remained after additionally adjusting for maternal pre-pregnancy BMI and maternal smoking during pregnancy. Previous studies have hypothesized that exposure to gestational diabetes may be a distal element driving the positive association of high birth weight and high fetal growth with CBT. ${ }^{4}$ However, the results from our study do not support this suggestion, since we found an inverse association that was independent of birth weight by gestational age. In further support of our findings, studies in adults have shown an inverse association between diabetes and risk of glioma. ${ }^{12,32}$ The similarity of our results and findings in adults is consistent with a shared etiology. ${ }^{33}$ An inverse association between diabetes and risk of glioma in adults may reflect a protective effect of increased immune surveillance in patients with autoimmune (type 1) diabetes. ${ }^{34,35}$ It is possible that such protection could be conferred to offspring in utero. However, a lower risk of CBT was seen among children of mothers with type 1 diabetes and children of mothers with gestational diabetes. Since gestational diabetes does not have a known autoimmunity component, our results suggest that a protective effect may be a consequence of hyperglycemia and/or hyperinsulinemia. ${ }^{36}$ In line with this, four of the nine genetic variants that predispose to insulin resistance or type 2 diabetes were also associated with a lower risk of neuroblastoma. Within these loci, LINC00824, PDX1-AS1, and especially KCNJ11 and $S Y N 2$ warrant further research to confirm or refute a causal effect on risk of neuroblastoma through glycaemia-related traits. $I G F 2$ is another obvious candidate gene, since in mice the autocrine action of this gene is required for beta-cell mass expansion during preganacy. ${ }^{37}$ However, the directions of effect observed for intronic variants in this gene imply its associations with neuroblastoma risk and type 1 diabetes antibodies represent at most genetic pleiotropy.

In support of previous studies, ${ }^{7,8}$ we found a $50 \%-60 \%$ higher incidence of leukemia and ALL among children whose mother had diabetes as compared with children of non-diabetic mothers. Birth weight and fetal growth, which are associated with maternal diabetes, have been identified as risk factors for childhood leukemia, potentially via IGF-1 pathways. ${ }^{5,6}$ However, we adjusted for birth weight by gestational age, and mechanisms independent of birth weight and fetal growth are thus more likely to explain our results. In analyses examining specific types of maternal diabetes, the largest effect sizes were observed for the associations of type 1 diabetes with leukemia and ALL. However, the association of type 1 diabetes and gestational diabetes with leukemia and ALL was directionally consistent, suggesting that hyperglycemia and/or hyperinsulinemia themselves are likely important. Of note, inference from results of analyses of diabetes subtype should be interpreted with caution, since the number of exposed cases was low.

This is the first study that has examined if subtypes of maternal diabetes are associated with risk of lymphoma. There were indications of an excess risk of lymphoma in children of mothers with type 2 diabetes and gestational diabetes, but not type 1 diabetes. There was no evidence of this association in additional analyses that included only the subset of the population with data on maternal pre-pregnancy BMI and maternal smoking during pregnancy. However, this subgroup contained few exposed cases.

The associations of maternal diabetes with CBT, leukemia, and ALL in offspring were stronger when children were followed until age 6 years, rather than age 15 years, and were attenuated by including follow-up to age 20 years. This indicates that maternal diabetes has the strongest effect on risk of childhood cancer in the earliest stages of life.

Strengths of this study are the national population-based cohort design and large sample size. Detailed information from national registers with high quality and validity enabled adjustment for potential confounding factors and reduced the chance of exposure misclassification and selection bias. However, since both the exposure and the outcomes addressed in this study are rare, the number of exposed cases is relatively low. In addition, some non-differential misclassification of diabetes status - where women with diabetes are not captured in the earlier periods of the study - is likely, since the inpatient register has only had national coverage since 1987, while data from the outpatient register are available only from 2001. As a result, the reported effects are conservative estimates, since some children who developed cancer may have had mothers with diabetes that was not captured. Some women classified as having type 2 diabetes may have an autoimmune form of diabetes, for which no separate ICD codes are available, for example latent autoimmune diabetes in adults; this may introduce further misclassification into the exposure. One further limitation is that only codes from ICD-7 were available throughout the follow-up period; so, it was not possible to classify childhood cancer according to the Birch and Marsden classification scheme. ${ }^{38}$ While this may limit comparability with studies that used different classification systems, it is unlikely to have a substantial impact on the results due to the broad disease subgroups included in this analysis. Although some children were excluded from 
analyses, $<1 \%$ of the whole cohort had missing data and so these exclusions are unlikely to influence our results.

Overall, the results of this study are compatible with a role of the prenatal and perinatal glycemic environments in the etiology of childhood cancer. Future studies with specific information on biomarkers and maternal glycemic control could provide additional insight into mechanisms that may underlie the association between diabetes and childhood cancer.

\section{Acknowledgments}

This work was supported by the Swedish Research Council for Health, Working Life and Welfare (FORTE) (grant registration number 2015-01228 to HLB) and Karolinska Institutet. MdH was supported by the Swedish Research Council (201503657), Swedish Heart-Lung Foundation (20140543) and NIH (R01DK106236 and R01DK107786). The funding bodies had no role in study design; in the collection, analysis, and interpretation of data; in the writing of the report; or in the decision to submit the article for publication.

\section{Disclosure}

The authors report no conflicts of interest in this work.

\section{References}

1. Kaatsch P. Epidemiology of childhood cancer. Cancer Treat Rev. 2010;36(4):277-285.

2. Johnson KJ, Cullen J, Barnholtz-Sloan JS, et al. Childhood brain tumor epidemiology: a brain tumor epidemiology consortium review. Cancer Epidemiol Biomarkers Prev. 2014;23(12):2716-2736.

3. Wiemels J. New insights into childhood leukemia etiology. Eur J Epidemiol. 2015;30(12):1225-1227.

4. Harder T, Plagemann A, Harder A. Birth weight and subsequent risk of childhood primary brain tumors: a meta-analysis. Am J Epidemiol. 2008;168(4):366-373.

5. Caughey RW, Michels KB. Birth weight and childhood leukemia: a meta-analysis and review of the current evidence. Int $J$ Cancer. 2009;124(11):2658-2670.

6. Milne E, Greenop KR, Metayer C, et al. Fetal growth and childhood acute lymphoblastic leukemia: findings from the childhood leukemia international consortium. Int J Cancer. 2013;133(12):2968-2979.

7. Contreras ZA, Ritz B, Virk J, Cockburn M, Heck JE. Maternal prepregnancy and gestational diabetes, obesity, gestational weight gain, and risk of cancer in young children: a population-based study in California. Cancer Causes Control. 2016;27(10):1273-1285.

8. Petridou E, Trichopoulos D, Kalapothaki V, et al. The risk profile of childhood leukaemia in Greece: a nationwide case-control study. Brit J Cancer. 1997;76(9):1241-1247.

9. Westbom L, Aberg A, Källén B. Childhood malignancy and maternal diabetes or other auto-immune disease during pregnancy. Br J Cancer. 2002;86(7):1078-1080.

10. Wu CS, Nohr EA, Bech BH, Vestergaard M, Olsen J. Long-term health outcomes in children born to mothers with diabetes: a population-based cohort study. PLoS One. 2012;7(5):e36727.

11. Carstensen B, Read SH, Friis S, et al; Diabetes and Cancer Research Consortium. Cancer incidence in persons with type 1 diabetes: a fivecountry study of 9,000 cancers in type 1 diabetic individuals. Diabetologia. 2016;59(5):980-988.
12. Zhao L, Zheng Z, Huang P. Diabetes mellitus and the risk of glioma: a meta-analysis. Oncotarget. 2016;7(4):4483-4489.

13. Vigneri R, Goldfine ID, Frittitta L. Insulin, insulin receptors, and cancer. J Endocrinol Invest. 2016;39(12):1365-1376.

14. Cnattingius S, Ericson A, Gunnarskog J, Källén B. A quality study of a medical birth registry. Scand J Soc Med. 1990;18(2):143-148.

15. Barlow L, Westergren K, Holmberg L, Talbäck M. The completeness of the Swedish Cancer Register: a sample survey for year 1998. Acta Oncol. 2009;48(1):27-33.

16. Lotta LA, Gulati P, Day FR, et al. Integrative genomic analysis implicates limited peripheral adipose storage capacity in the pathogenesis of human insulin resistance. Nat Genet. 2017;49(1):17-26.

17. Plagnol V, Howson JM, Smyth DJ, et al; Type 1 Diabetes Genetics Consortium. Genome-wide association analysis of autoantibody positivity in type 1 diabetes cases. PLoS Genet. 2011;7(8):e1002216.

18. DIAbetes Genetics Replication And Meta-analysis (DIAGRAM) consortium; Asian Genetic Epidemiology Network Type 2 Diabetes (AGEN-T2D) Consortium; South Asian Type 2 Diabetes (SAT2D) Consortium; Mexican American Type 2 Diabetes (MAT2D) Consortium; Type 2 Diabetes Genetic Exploration by Nex-generation sequencing in muylti-Ethnic Samples (T2D-GENES) Consortium, Mahajan A, Go MJ, Zhang W, et al. Genome-wide trans-ancestry meta-analysis provides insight into the genetic architecture of type 2 diabetes susceptibility. Nat Genet. 2014;46(3):234-244.

19. Manning AK, Hivert MF, Scott RA, et al. A genome-wide approach accounting for body mass index identifies genetic variants influencing fasting glycemic traits and insulin resistance. Nat Genet. 2012;44(6):659-669.

20. Wang K, Diskin SJ, Zhang H, et al. Integrative genomics identifies LMO1 as a neuroblastoma oncogene. Nature. 2011;469(7329):216-220.

21. Urayama KY, Jarrett RF, Hjalgrim H, et al. Genome-wide association study of classical Hodgkin lymphoma and Epstein-Barr virus statusdefined subgroups. J Natl Cancer Inst. 2012;104(3):240-253.

22. Han S, Lee KM, Park SK, et al. Genome-wide association study of childhood acute lymphoblastic leukemia in Korea. Leuk Res. 2010;34(10): 1271-1274.

23. Welter D, MacArthur J, Morales J, et al. The NHGRI GWAS Catalog, a curated resource of SNP-trait associations. Nucleic Acids Res. 2014;42(Database issue):D1001-D1006.

24. Arnold M, Raffler J, Pfeufer A, Suhre K, Kastenmüller G. SNiPA: an interactive, genetic variant-centered annotation browser. Bioinformatics. 2015;31(8):1334-1336.

25. Leslie R, O'Donnell CJ, Johnson AD. GRASP: analysis of genotypephenotype results from 1390 genome-wide association studies and corresponding open access database. Bioinformatics. 2014;30(12):i185-i194.

26. Boyle AP, Hong EL, Hariharan M, et al. Annotation of functional variation in personal genomes using RegulomeDB. Genome Res. 2012;22(9):1790-1797.

27. GTEx Consortium; Lonsdale J, Thomas J, Salvatore M, et al. The Genotype-Tissue Expression (GTEx) project. Nat Genet. 2013;45(6): 580-585.

28. Yong PF, Freeman AF, Engelhardt KR, Holland S, Puck JM, Grimbacher B. An update on the hyper-IgE syndromes. Arthritis Res Ther. 2012;14(6):228.

29. Woellner C, Gertz EM, Schäffer AA, et al. Mutations in STAT3 and diagnostic guidelines for hyper-IgE syndrome. JAllergy Clin Immunol. 2010;125(2):424-432.e8.

30. Ellis AK, Waserman S. Hodgkin's lymphoma presenting with markedly elevated IgE: a case report. Allergy Asthma Clin Immunol. 2009;5(1):12.

31. Stevanović S, van Schie ML, Griffioen M, Falkenburg JH. HLA-class II disparity is necessary for effective $\mathrm{T}$ cell mediated Graft-versusLeukemia effects in NOD/scid mice engrafted with human acute lymphoblastic leukemia. Leukemia. 2013;27(4):985-987.

32. Kitahara CM, Linet MS, Brenner AV, et al. Personal history of diabetes, genetic susceptibility to diabetes, and risk of brain glioma: a pooled analysis of observational studies. Cancer Epidemiol Biomarkers Prev. 2014;23(1):47-54. 
33. Adel Fahmideh M, Lavebratt C, Schüz J, et al. CCDC26, CDKN2BAS, RTEL1 and TERT Polymorphisms in pediatric brain tumor susceptibility. Carcinogenesis. 2015;36(8):876-882.

34. Markiewicz MA, Gajewski TF. The immune system as anti-tumor sentinel: molecular requirements for an anti-tumor immune response. Crit Rev Oncog. 1999;10(3):247-260.

35. Pardoll D. Does the immune system see tumors as foreign or self? Annu Rev Immunol. 2003;21:807-839.
36. Seliger $\mathrm{C}$, Ricci $\mathrm{C}$, Meier CR, et al. Diabetes, use of antidiabetic drugs, and the risk of glioma. Neuro-Oncol. 2016;18(3):340-349.

37. Modi H, Jacovetti C, Tarussio D, et al. Autocrine action of IGF2 regulates adult $\beta$-cell mass and function. Diabetes. 2015;64(12): $4148-4157$.

38. Birch JM, Marsden HB. A classification scheme for childhood cancer. Int J Cancer. 1987;40(5):620-624.

\section{Publish your work in this journal}

Clinical Epidemiology is an international, peer-reviewed, open access, online journal focusing on disease and drug epidemiology, identification of risk factors and screening procedures to develop optimal preventative initiatives and programs. Specific topics include: diagnosis, prognosis, treatment, screening, prevention, risk factor modification,

Submit your manuscript here: https://www.dovepress.com/clinical-epidemiology-journal
Dovepress

systematic reviews, risk and safety of medical interventions, epidemiology and biostatistical methods, and evaluation of guidelines, translational medicine, health policies and economic evaluations. The manuscript management system is completely online and includes a very quick and fair peer-review system, which is all easy to use. 